\title{
Energy Levels of Helium Nucleus
}

\author{
Cvavb Chandra Raju \\ Department of Physics, Osmania University, Hyderabad, India \\ Email: cvavbc@gmail.com
}

Received January 9, 2013; revised February 10, 2013; accepted February 22, 2013

Copyright (C) 2013 Cvavb Chandra Raju. This is an open access article distributed under the Creative Commons Attribution License, which permits unrestricted use, distribution, and reproduction in any medium, provided the original work is properly cited.

\begin{abstract}
The Helium-4 nucleus is more similar to the Hydrogen atom of atomic physics. In the case of hydrogen atom, there are many energy levels which were experimentally seen and theoretically explained using non-relativistic quantum mechanics. In this note, we use a central potential to derive the energy levels of Helium- 4 nucleus. The ground state and the first few energy levels agree pretty well with experiment. The same potential can be used with nuclei like Oxygen-17 and many more nuclei.
\end{abstract}

Keywords: Helium-4; Nuclear Energy Levels; Deuteron; Morphed Gravitational Potential

\section{Introduction and Formulation of the Problem}

The Deuteron nucleus has no excited states. The ground state energy of the Deuteron is experimentally found to be $-2.225 \mathrm{MeV}$ and the measured radius of this nucleus is $2.1 \mathrm{~F}$. This is the distance between the center of mass and either of the nucleon in the Deuteron nucleus [1]. There are no known solutions of the Schrödinger equation of this nucleus with Yukawa potential.

Are there any central potentials with which we can solve the Schrödinger equation for many nuclei such that their ground state wave functions and excited states can be obtained? This question led us to a central potential which is closely related to the gravitational potential energy.

There is no reason or experimental support to believe that the universal constant of gravitation $G$ is same for all values of interacting masses. For interacting masses of the order of nucleon masses $G$ may not retain its universality. This led us to the following expression for the gravitational potential energy of two particles whose masses are $m_{1}$ and $m_{2}$,

$$
V(r)=-G\left[1-\mathrm{e}^{-\frac{g^{2} \hbar c}{G M_{0}^{2}}}\right] \frac{m_{1} m_{2}}{r},
$$

where in place of the usual constant of universal constant $G$ we have a modulating factor.

The constant $g^{2}$ is a dimensionless real number whereas $M_{0}$ has dimensions of mass. We believe that the expo- nential goes to zero when the interacting masses are large and the Universal law of Gravitation is restored. It is the parameter $M_{0}$ that causes the Universal Law of Gravitation restored. An approximation to Equation (1.1) is given by,

$$
V(r)=-G\left[1-\left\{1-\frac{g^{2} \hbar c}{G M_{0}^{2}}\right\}\right] \frac{m_{1} m_{2}}{r},
$$

where " $r$ " is the distance between the interacting particles. Simplifying Equation (1.2), we have,

$$
V(r)=-\frac{g^{2} \hbar c}{M_{0}^{2}} \frac{m_{1} m_{2}}{r} .
$$

The above potential energy is obtained from the gravitational potential energy. It may be called "the morphed gravitational potential energy". There are two constants $g^{2}$ and $M_{0}^{2}$ which we will obtain below.

\section{Deuteron}

The Deuteron is a bound system of a neutron and a proton with an orbital angular momentum of zero. The total spin of the two nucleons is one. The deuteron nucleus has no excited states. The experimentally measured ground state energy of the Deuteron nucleus is $-2.225 \mathrm{MeV}$ and its orbital angular quantum number $\ell=0$. There is no stable diproton. It is also known that the nuclear potential depends on the spin orientation [2] of the nucleons inside a nucleus. If the nuclear force is independent of their spin orientation then the singlet (total spin $=0$ ) state and the triplet state (total spin $=1$ ) will have the same energy. 
But this is not observed. This means that the singlet tensor potential is weaker than the triplet potential. We consider an extreme situation wherein the singlet potential of the diproton is quite negligible. In that event the total potential energy operating for a diproton nucleus is,

$$
-\frac{g^{2} \hbar c m_{p}^{2}}{M_{0}^{2} r}+\frac{e^{2} \hbar c}{r},
$$

where, the first term is the morphed gravitational potential energy and the second term is the Coulomb potential energy between the two protons. Here $e^{2}=\frac{1}{137}$ is the fine structure constant. If, $M_{0}^{2}=m_{p}^{2}$ and $g^{2}=e^{2}$, the total potential energy in the case of a diproton will be zero and there will be no stable diproton nucleus! But with these values for $g^{2}$ and $M_{0}^{2}$ the theoretically computed binding energy for the deuteron nucleus turns out to be quite small. Hence we chose the following values for these parameters which are quite close to the values mentioned above.

$$
g^{2}=\frac{e^{2}}{0.2254}=0.032384,
$$

and,

$$
M_{0}^{2}=0.931826 \times 10^{-48}(\mathrm{gm})^{2} .
$$

There are a few important points to note about the above values of the parameters $g^{2}$ and $M_{0}^{2}$.

- The parameter $g^{2}$ is the weak interaction constant of the electro-weak standard model [3] because it contains $e^{2}$ and the Weinberg mixing parameter $\sin ^{2} \theta_{W}=$ 0.2254 as in the Standard model [4]. The other parameter is nearly equal to the square of the proton mass and this is required to avoid a stable diproton nucleus.

- The interaction parameter contains the square of mass in the numerator and also in the denominator; Because of this reason the interaction constant of the morphed gravitational potential has the same dimensions as the electroweak constant. Hence it is renormalizable. This is an important point in favor of the morphed gravitational potential.

The Schrödinger equation for the deuteron is given by,

$$
\left[-\frac{\hbar^{2}}{2 \mu} \nabla^{2}+V(r)\right] \psi(r, \theta, \phi)=E \psi(r, \theta, \phi),
$$

where, $V(r)$ is given by Equation (1.3) with $m_{1}=m_{p}$ and $m_{2}=m_{n}$. The reduced mass $\mu$ is given by,

$$
\mu=\frac{m_{p} m_{n}}{m_{p}+m_{n}} .
$$

Since $V(r)$ is a central potential, from the methods of quantum mechanics, it just follows that $[5,6]$,

$$
\psi(r, \theta, \phi)=R_{n \ell}(r) Y_{\ell m}(\theta, \phi) .
$$

As in the case of the Hydrogenatom, the quantum numbers, $n, \quad \ell$ and $m$ have their values,

$$
\begin{aligned}
& n=1,2,3, \cdots, \ell=0,1, \cdots, n-1 \text { and } \\
& m=\ell, \ell-1, \ell-2, \cdots,-\ell
\end{aligned}
$$

The radial function is given by,

$$
R_{n \ell}=A \mathrm{e}^{-\frac{\rho}{2}} \rho^{\ell} L_{(n+\ell)}^{2 \ell+1}(\rho)
$$

where,

$$
\rho=\frac{2 r}{n a_{0}}
$$

and,

$$
a_{0}=\frac{\hbar^{2}}{\mu} \frac{M_{0}^{2}}{\left(g^{2} \hbar c\right) m_{p} m_{n}} .
$$

These results are obtained by a simple transcription of the Hydrogenatom calculations. The energy of the deuteron is given by,

$$
E_{n \ell}=-\frac{\mu}{2 \hbar^{2}}\left(\frac{g^{2} \hbar c}{M_{0}^{2}}\right)^{2} \frac{\left(m_{p} m_{n}\right)^{2}}{n^{2}} .
$$

The normalization constant $A$ in Equation (2.8) is given by,

$$
A=\sqrt{\left(\frac{2}{n a_{0}}\right)^{3}} \sqrt{\frac{1}{2 n}} \sqrt{\left\{\frac{(n-\ell-1) !}{[(n+\ell) !]^{3}}\right\}} .
$$

Using the above results we can now estimate the ground state energy, ground state wave function and the radius of the Deuteron nucleus.

$$
\begin{gathered}
E_{10}=-2.2251 \mathrm{MeV} . \\
\psi_{100}=\frac{1}{\sqrt{\pi a_{0}^{3}}} \mathrm{e}^{-\frac{r}{a_{0}}} . \\
a_{0}=4.31734 \times 10^{-13} \mathrm{~cm} .
\end{gathered}
$$

here $a_{0}$ is the distance between the two nucleons. The radius of the Deuteron is the distance of either of the nucleon from the center of mass. The center of mass lies halfway between the line joining the two nucleons because the two nucleons have almost equal mass. We cannot avoid the excited states of the deuteron nucleus however small these may be.

\section{The Helium-4 Nucleus}

The Helium-4 nucleus is a strongly bound system of two neutrons and two protons. It used to be known as an $\alpha$ particle. In fact using this $\alpha$-particle itself the very ex- 
istence of a nucleus was inferred. Our morphed potential energy can be put to test using the estimated energy levels for this nucleus. A rough picture of this nucleus is like this. Any one nucleon experiences a total morphed gravitational potential due to the remaining three nucleons. This outer nucleon must be a proton because two protons of this nucleus cannot be together because of their Coulomb repulsion. This rough picture indicates that,

$$
V(r)=-\frac{g^{2} \hbar c}{M_{0}^{2}} \frac{\left(2 m_{n}+m_{p}\right) m_{p}}{r}+\frac{e^{2} \hbar c}{r},
$$

where the second term is due to the Coulomb repulsion, and the first term is the morphed gravitational potential energy. We will now apply the methods of quantum mechanics in an effort to obtain a theoretical description of Helium-4 nucleus or alpha-particle.

The Schrödinger equation with the above potential is given by

$$
\left[-\frac{\hbar^{2}}{2 \mu} \nabla^{2}+V(r)\right] \psi(r, \theta, \phi)=E \psi(r, \theta, \phi),
$$

where the reduced mass is now given by,

$$
\mu=\frac{\left(2 m_{n}+m_{p}\right) m_{p}}{\left(2 m_{n}+2 m_{p}\right)}=1.254749 \times 10^{-24} \mathrm{gm} .
$$

The central potential $V(r)$ enables us to find a closed solution for this nucleus. As in the case of the H-atom the radial function is given by,

$$
R_{n \ell}=A \mathrm{e}^{-\frac{\rho}{2}} \rho^{\ell} L_{(n+\ell)}^{2 \ell+1}(\rho)
$$

where,

$$
\rho=\frac{2 r}{n a_{0}}
$$

here,

$$
a_{0}=\frac{\hbar^{2}}{\mu} \frac{1}{\left[\frac{g^{2} \hbar c\left(2 m_{n}+m_{p}\right) m_{p}}{M_{0}^{2}}-e^{2} \hbar c\right]} .
$$

In Equations (3.4) and (3.5), $n$ is the principal quantum number as in Equation (2.7). The energy Eigen values for the Helium nucleus are given by,

$$
E=-\frac{\mu}{2 \hbar^{2}} \frac{\left[\frac{g^{2} \hbar c\left(2 m_{n}+m_{p}\right) m_{p}}{M_{0}^{2}}-e^{2} \hbar c\right]^{2}}{n^{2}} .
$$

The normalization constant $A$ in Equation (3.4) is given by,

$$
A=\sqrt{\left(\frac{2}{n a_{0}}\right)^{3}} \sqrt{\frac{1}{2 n}} \sqrt{\left\{\frac{(n-\ell-1) !}{[(n+\ell) !]^{3}}\right\}} .
$$

For the Helium nucleus the ground state orbital angular momentum quantum number is zero. Hence the ground state wave function of the Helium- 4 nucleus is given by,

$$
\psi_{100}=\frac{1}{\sqrt{\pi a_{0}^{3}}} \mathrm{e}^{-\frac{r}{a_{0}}} .
$$

The ground state energy of Helium- 4 nucleus is given by,

$$
E_{10}=-28.5176 \mathrm{MeV} \text {. }
$$

This value should be compared with the binding energy of this nucleus which is about $28.3 \mathrm{MeV}$; we obtained this value through ordinary quantum mechanics and by using masses and the charge as shown above. In general the principal energy levels of the tightly bound Helium-4 nucleus are given by,

$$
E_{n}=-\frac{28.5176}{n^{2}} \mathrm{MeV} \text {. }
$$

The possible energies for this nucleus are listed below:

$$
\begin{array}{ll}
E_{1}=-28.5176 \mathrm{MeV}, & E_{2}=-7.1294 \mathrm{MeV}, \\
E_{3}=-3.168622 \mathrm{MeV}, & E_{4}=-1.78235 \mathrm{MeV}, \quad(3.12) \\
E_{5}=-1.140704 \mathrm{MeV}, & E_{6}=-0.792156 \mathrm{MeV} .
\end{array}
$$

These energy levels are obtained ignoring the tensor forces. These energy levels can be put to test to find out the viability of the morphed gravitational potential. Our efforts to find the principal energy levels were not very successful. In reference [7], only we could obtain the measured energy spectrum and this contains only two energy levels. The energy diagram is arranged by setting the ground state energy zero, this amount to adding $+28.5176 \mathrm{MeV}$ to all the energies above. Our results agree pretty well with the two principal levels given in Ref [7]. The general wave function for the Helium-4 nucleus for any orbital angular momentum is given by,

$$
\psi(r, \theta, \phi)=R_{n \ell}(r) Y_{\ell m}(\theta, \phi) .
$$

The wave function given here for the ground state of the Helium-4 nucleus can be used in alpha-decay or in reactions involving the alpha particle.

\section{Conclusion}

In this note we assumed that the universal constant $G$ is not universal for all values of the interacting masses. Motivated by this idea we changed $G$ and used an approximated constant for interacting nucleons. When this potential is applied along with the methods of ordinary 
quantum mechanics the estimated results are all in agreement with experiment. This very procedure can be applied to such nuclei Oxygen-17, F-17 and many more nuclei. The constant $M_{0}^{2}$ is not universal. It is an adjustable parameter. The functional dependence of this parameter on the product of the interacting masses is unclear as of now. But the idea cannot be dismissed just like that. But certainly for larger masses the exponential in Equation (1.1) goes to zero.

\section{Acknowledgements}

The author is very grateful to Prudhvi Rchintalapati. But for his help the work could not have seen the light of the day.

\section{REFERENCES}

[1] R. J. Bin-Stoyle, "Nuclear and Particle Physics," Chapman and Hall, Madras, 1991. doi:10.1007/978-94-010-9561-7

[2] H. A. Enge, "Introduction to Nuclear Physics," AddisonWesley, Ontario, 1966.

[3] S. Weinberg, "A Model of Leptons," Physical Review Letters, Vol. 19, No. 21, 1967, pp. 1264-1266.

[4] C. C. Raju, "Majorana Mass of the Electron-Muon Mass of the Dirac Neutrino and Fermion Masses," International Journal of Theoretical Physics, Vol. 36, No. 12, 1997, pp. 2937-2935.

[5] L. I. Schiff, "Quantum Mechanics," Mcgraw-Hill, New York, 1955.

[6] S. Gasiorowicz, "Quantum Physics," Addison-Wesley, Boston, 1974.

[7] R. Telley and H. R. Weller, "Energy Levels of Light Nuclei," Nuclear Physics A, Vol. 141, 1992, p. 1. 